\title{
Measurement of linear coefficient of thermal expansion and temperature-dependent refractive index using interferometric system
}

\author{
James A. Corsetti, ${ }^{1, *}$ William E. Green, ${ }^{1,2}$ Jonathan D. Ellis, ${ }^{1,2}$ Greg R. Schmidt, ${ }^{1}$ and Duncan T. Moore, ${ }^{1}$ \\ ${ }^{1}$ The Institute of Optics, University of Rochester, Wilmot Building, 275 Hutchison Road, Rochester, NY 14627, USA \\ ${ }^{2}$ Department of Mechanical Engineering, University of Rochester, 235 Hopeman Building, Box 270132, Rochester, NY 14627, USA \\ * jcorset2@u.rochester.edu
}

\begin{abstract}
A system combining an interferometer with an environmental chamber for measuring both coefficient of thermal expansion (CTE) and temperature-dependent refractive index $(\mathrm{dn} / \mathrm{dT})$ simultaneously is presented. The operation and measurement results of this instrument are discussed.

OCIS codes: (120.6810) Thermal effects; (120.3180) Interferometry; (120.3940) Metrology
\end{abstract}

\section{Introduction}

The thermal response of an optical element is described mathematically by a number of material parameters. First amongst these is the linear coefficient of thermal expansion (CTE). As a material, optical or otherwise, is heated or cooled, it changes its physical size in keeping with this value. This is represented mathematically by

$$
L^{\prime}=L(1+\alpha \Delta T) \text {, }
$$

where $L$ and $L^{\prime}$ are length of the sample before and after a temperature change of $\Delta T$ while $\alpha$ is the CTE [1]. Thus, exposing a material with a positive CTE (as the vast majority do have) to a temperature increase will cause that sample to expand in each dimension.

The second thermal parameter of interest is the temperature-dependent refractive index $(\mathrm{dn} / \mathrm{dT})$. This value determines how the index of refraction of an element will change with a given $\Delta T$ and is represented mathematically according to

$$
N_{00}^{\prime}=N_{00}+\left(\frac{d n}{d T}\right) \Delta T,
$$

where $N_{O 0}$ and $N_{O O}$ ' are the sample's index of refraction before and after a temperature change of $\Delta T$, respectively [2]. While $\mathrm{dn} / \mathrm{dT}$ is most often a positive value, for certain materials such as $\mathrm{CaF}_{2}$ and various polymers, these values are negative. Note that each of these parameters call back to one of the two quantities that make up the expression for optical path: physical thickness and index of refraction.

Knowledge of both the CTE and dn/dT values of all materials present in an optical design is necessary to determine how that system will behave when exposed to a varying environment. Under certain circumstances, a lens system can be passively athermalized so that no focus adjustment is required for different operating temperatures; otherwise, active athermalization is accomplished through the use of a moving compensator, most likely the detector plane. Traditionally, CTE has been measured with a dilatometer while $\mathrm{dn} / \mathrm{dT}$ can be determined using a refractometer to measure index at different temperatures and taking the derivative with respect to temperature to find $\mathrm{dn} / \mathrm{dT}$. This paper explores means of measuring these quantities interferometrically.

\section{Measurement process}

Previously, a number of institutions have demonstrated the ability to measure CTE and dn/dT individually and together interferometrically [3-5]. Most commonly, the interferometer is built so that both the test and reference arms of are subjected to the same environment. Additionally, the measurements are often in vacuum. In this way, the measured optical path difference is not affected at all by the refractive index of air changing with temperature. However, the vacuum pressure also distorts the mechanical size of the optic, which will ultimately affect the measured CTE and dn/dT values. This paper explores a method to measure both CTE and dn/dT simultaneously with the two arms of the interferometer in different environments.

Fabry-Perot thermal systems have previously been reported [6-7]. A Twyman-Green configuration was instead chosen for this work because of its reduced cost and added benefit of not having differing cavity finesse when used to measure with different wavelengths [8]. The tradeoff to this, when compared to the Fabry-Perot, is that the limited size of the Espec BTX-475 environmental chamber being used requires the reference arm be outside of the 
environmental chamber while the test arm down extends inside of it. The resulting interferograms are sensitive to mechanical vibration and thermal drift. A photograph and diagram of the system are shown in Figure 1.
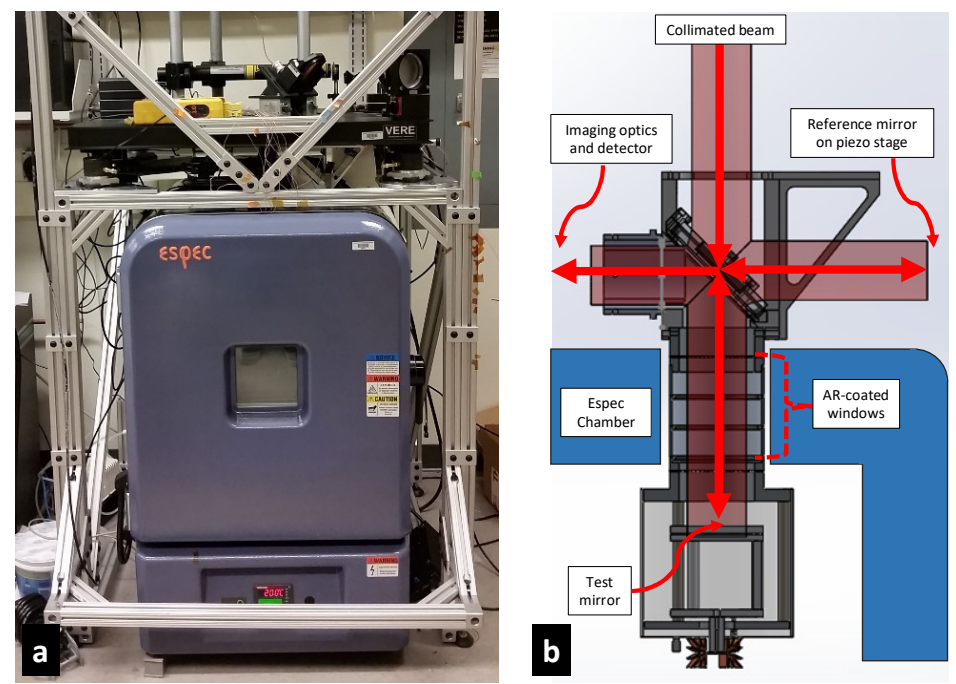

Figure 1: (a) Photograph of thermal interferometer above environmental chamber. (b) Schematic of interferometer system

Measurements are performed by placing a sample, half-coated with gold or another reflective material, atop the test mirror in the interferometer. This causes three separate interferograms to form between the reference mirror and (1) the test mirror, $d O P D_{1},(2)$ the reflection from the top of the sample, $d O P D_{2}$, and (3) the reflection from the test mirror having transmitted through the sample, $d O P D_{3}$. These three beam paths are indicated in Figure 2.

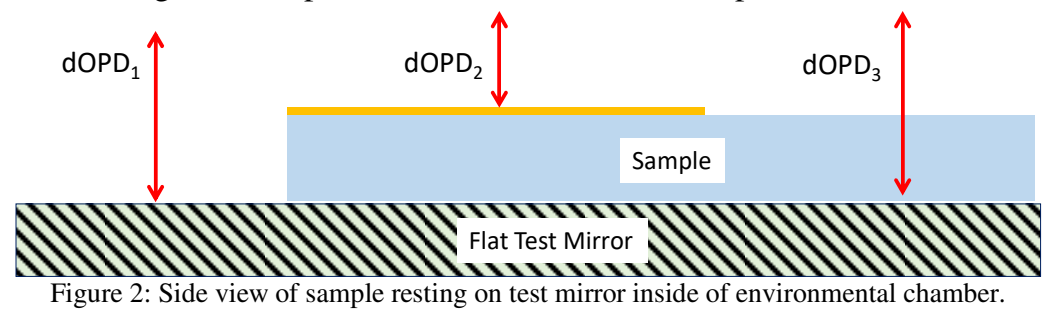

The reference mirror is mounted on a piezo stage, making the system capable of phase-shifting. As the temperature is cycled over the range of interest, phase-maps are continuously generated. An example is shown in Figure 3 where a coated sample is seen side-by-side with a computed phasemap. By unwrapping these phasemaps as a function of time and therefore temperature, one can determine the accumulated piston over the course of the measurement for each pixel in each of the three regions of interest. Comparing the amounts of piston accumulated in paths $d O P D_{I}$ and $d O P D_{2}$ to one another, the CTE of the sample is determined while the addition of path $d O P D_{3}$ yields $\mathrm{dn} / \mathrm{dT}$.
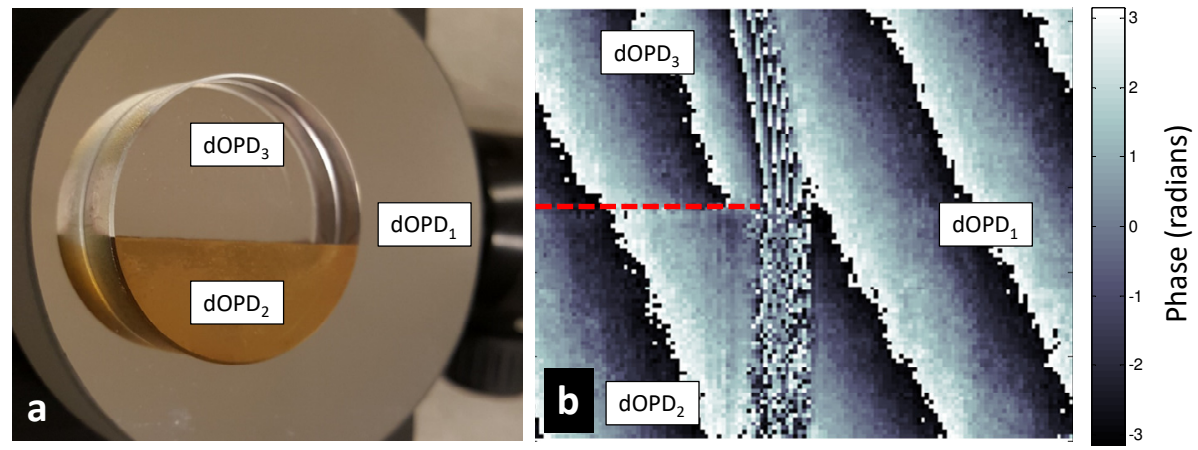

Figure 3. (a) Photograph of sample half-coated with gold resting on test mirror for measurement (b) Computed phase-map. Both (a) and (b) show three beam paths of interest for determining CTE and dn/dT

\section{Results}

A number of optical materials have previously been measured using the instrument including steel and $\mathrm{CaF}_{2}[8]$. Additional measurements on the CTE and dn/dT of the polymers polymethyl methacrylate (PMMA) and polystyrene 
at a wavelength of $632.8 \mathrm{~nm}$ have been carried out between 5 and $35{ }^{\circ} \mathrm{C}$ as shown in Figure 4 . The results are consistent with the large range of values reported by other sources for these polymers [9-12].
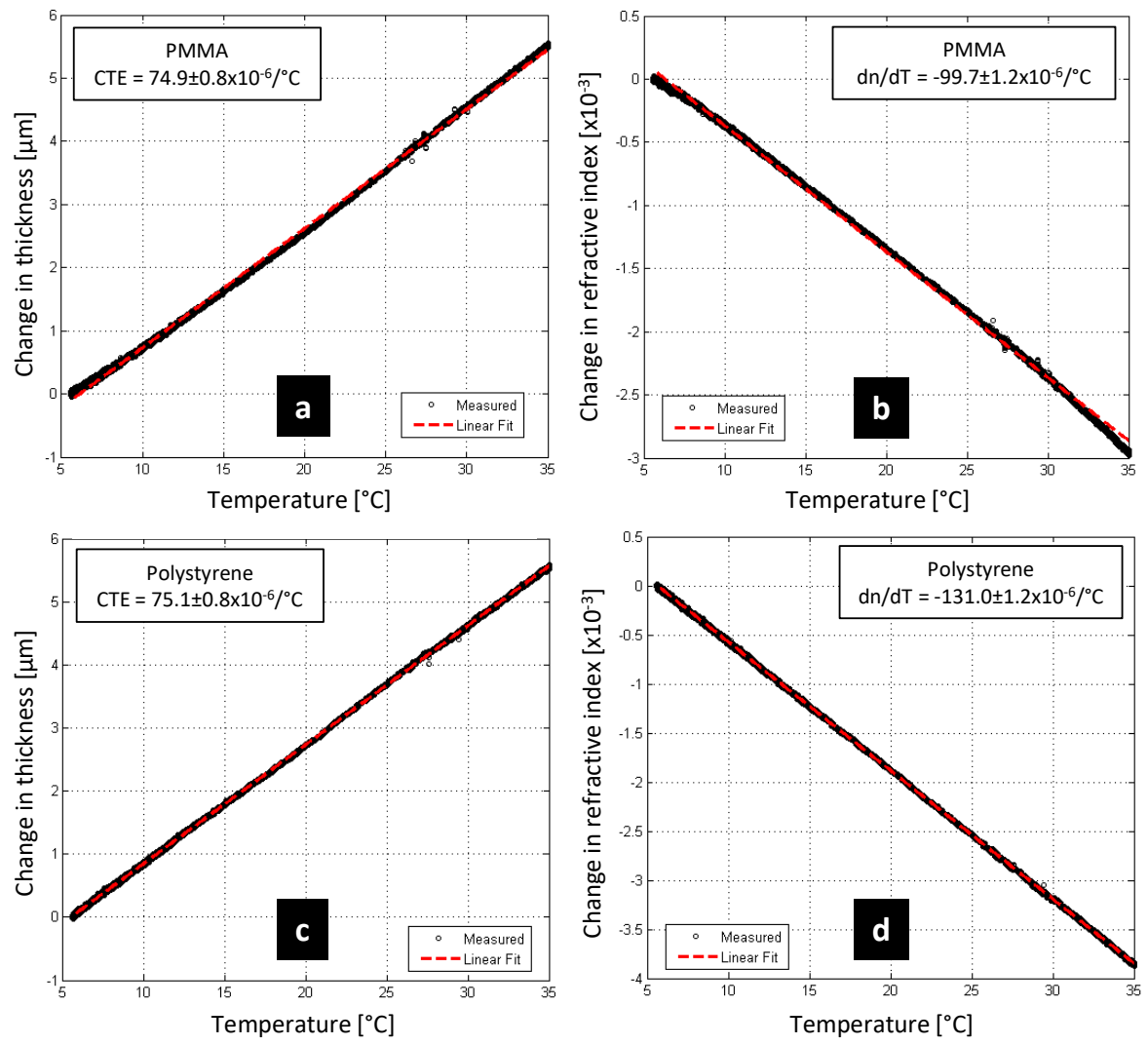

Figure 4: Measured change in (a) thickness and (b) index of refraction for PMMA sample $\left(t_{0}=2.522 \mathrm{~mm}, n_{0}=1.490\right)$. Measured change in (c) thickness and (d) index of refraction for polystyrene sample $\left(t_{0}=2.508 \mathrm{~mm}, n_{0}=1.585\right)$.

\section{References}

[1] K. B. Doyle, V. L. Genberg, and G. J. Michels, "Optothermal Analysis Methods," in Integrated Optomechanical Analysis (SPIE Press, Bellingham, WA, 2012), pp. 279-298.

[2] T. H. Jamieson, "Thermal Effects in Optical Systems," Optical Engineering 20, 156-160 (1981).

[3] J. D. James, J. A. Spittle, S. G. R. Brown, and R. W. Evans, "A review of measurement techniques for the thermal expansion coefficient of metals and alloys at elevated temperatures," Measurement Science and Technology 12, R1-R15 (2001).

[4] M. Okaji, N. Yamada, and H. Moriyama, "Ultra-precise thermal expansion measurements of ceramic and steel gauge blocks with an interferometric dilatometer," Metrologia 37, 165-171 (2000)

[5] J. M. Jewell, C. Askins, and I. D. Aggarwal, "Interferometric method for concurrent measurement of thermo-optic and thermal expansion coefficients," Appl. Opt. 30, 3656-3660 (1991).

[6] P. O. McLaughlin and D. T. Moore, "Measurement of the differential thermal expansion and temperature dependence of refractive index in gradientindex glass," Appl. Opt. 24, 4334-4341 (1985).

[7] P. McCarthy, J. Corsetti, D. T. Moore, and G. R. Schmidt, "Application of a Multiple Cavity Fabry-Perot Interferometer for Measuring the Thermal Expansion and Temperature Dependence of Refractive Index in New Gradient-Index Materials," in Imaging and Applied Optics Technical Papers, OSA Technical Digest Series (Optical Society of America, 2012), paper OTu2D.3.

[8] J. A. Corsetti, W. E. Green, J. D. Ellis, G. R. Schmidt, and D. T. Moore, "Simultaneous interferometric measurement of linear coefficient of thermal expansion and temperature-dependent refractive index coefficient of optical materials," Appl. Opt. 55, 8145-8152 (2016)

[9] “Thermal Properties,” http://www.m-ep.co.jp/en/pdf/product/iupi_nova/physicality_04.pdf

[10] "Properties of Optical Plastic Materials," http://www.plasticoptics.com/optical-plastic-materials.html

[11] The Handbook of Plastic Optics. 2 ed. 1983, Cincinatti: U.S. Precision Lens.

[12] "Plastic Optics: Specifying Injection-Molded Polymer Optics," https://www.photonics.com/EDU/Handbook.aspx?AID=25487 